\title{
Object continuity and the transsaccadic representation of form
}

\author{
Robert D. Gordon, Sarah D. Vollmer, and Megan L. Frankl \\ North Dakota State University, Fargo, North Dakota
}

\begin{abstract}
Transsaccadic object file representations were investigated in three experiments. Subjects moved their eyes from a central fixation cross to a location between two peripheral objects. During the saccade, this preview display was replaced with a target display containing a single object to be named. On trials on which the target identity matched one of the preview objects, its orientation either matched or did not match the previewed orientation. The results of Experiments 1 and 2 revealed that orientation changes disrupt perceptual continuity for objects located near fixation, but not for objects located further from fixation. The results of Experiment 3 confirmed that orientation changes do not disrupt continuity for distant objects, while showing that subjects nevertheless maintain an object-specific representation of the orientation of such objects. Together, the results suggest that object files represent orientation but that whether or not orientation plays a role in the processes that determine continuity depends on the quality of the perceptual representation.
\end{abstract}

While viewing a scene, the eyes do not remain stationary. Instead, saccadic eye movements occur two to three times per second, interrupted by brief fixations during which new information about the scene is acquired. Because vision is severely impaired during saccades (Matin, 1974), the input to the visual system is provided primarily by the fixations and, therefore, consists of a series of "snapshots" of the scene. In order to arrive at a full understanding of the scene content, it is necessary to combine information across saccades. In the present study, we examine the process of representing and integrating information across saccades.

Although some early studies suggested that transsaccadic integration might rely on a high-capacity representation (e.g., Feldman, 1985; McConkie \& Rayner, 1976), more recent evidence suggests that transsaccadic memory is actually quite limited (e.g., Irwin, 1991, 1992; Irwin \& Andrews, 1996; O'Regan, 1992). Irwin and Andrews, for example, studied transsaccadic memory using a partial report task. In their experiment, subjects began each trial by viewing a central fixation cross. An array of 6 or 10 colored letters was then presented, along with a peripheral saccade target to which the subjects were directed to move their eyes. During the saccade to the new target, the letters were removed; when the eyes landed, one of the array locations was probed, and the subjects' task was to report the identity and color of the letter that had appeared in that location. Contrary to what would be expected if transsaccadic memory had a very high capacity, Irwin and Andrews found that the subjects could report the color and identity of only 3-4 of the letters in the array. Interestingly, this capacity was very similar to that reported by Irwin (1992), who required subjects to report letter identity alone. Irwin and Andrews concluded that transsaccadic memory consists primarily of integrated object representations (which may include a number of object features), along with residual activity in the feature maps that underlie sensory processing. Subsequent work in which more complex stimuli were used also suggests that transsaccadic memory consists primarily of representations of a small number of objects in the scene (e.g., Irwin \& Zelinsky, 2002).

A representation of the sort thought to make up transsaccadic memory is the object file proposed by Kahneman and Treisman (1984; Kahneman, Treisman, \& Gibbs, 1992). Indeed, Irwin and his colleagues have described their account as an "object file theory of transsaccadic memory" (e.g., Irwin, 1996; Irwin \& Andrews, 1996; Irwin \& Gordon, 1998), and others have similarly proposed that representations of object tokens underlie much of transsaccadic memory (e.g., Henderson, 1994; Henderson \& Anes, 1994). As described by Kahneman et al., object files are temporary episodic representations of specific objects in the visual field. According to object file theory, object files represent the features of a particular object at a specific location and time. This may include an object's perceptual features but may also include nonperceptual features, such as an object's identity, if such information can be acquired from the image (e.g., Gordon \& Irwin, 1996, 2000). An important property of object file representations, therefore, is that they are relatively dynamic, subject to change as new information is acquired.

According to object file theory, object files play a critical role in preserving continuity within and across

R. D. Gordon, robert.d.gordon@ndsu.edu 
fixations. On this account, continuity depends on the outcome of a series of processing stages. During an encoding stage, representations are formed for a limited number of attended objects within the environment. When there is a change in the environment (whether through movement of objects, movement of the observer, or movement of the eyes), a correspondence process establishes a link between the currently visible objects and the stored object files. This correspondence has been proposed to rely exclusively on relative object location (Henderson, 1994; Henderson \& Anes, 1994; Kahneman et al., 1992), although more recent evidence suggests that other object features (such as color or even identity) may, under some circumstances, play a significant role in establishing correspondence across views (Richard, Hollingworth, \& Luck, 2006).

Following the establishment of a correspondence between visible objects and stored object files, a reviewing process is engaged. This process consists of the retrieval of an object file and a comparison of its features with the features of the corresponding object. Continuity is thought to rely critically on the outcome of that comparison. If the stored representation matches the corresponding object's features, continuity is preserved. If, on the other hand, there is a mismatch between the object and its representation, continuity is disrupted. Under such circumstances, it is necessary to construct a new object file to represent the object; because this process is believed to take time, subsequent processing of the object is delayed.

Kahneman et al. (1992) developed a new experimental paradigm (often referred to as the reviewing paradigm) for studying episodic object representation. Their approach has been very influential in subsequent work on object files (e.g., Gajewski \& Henderson, 2005; Germeys, De Graef, \& Verfaillie, 2002; Gordon \& Irwin, 1996, 2000; Noles, Scholl, \& Mitroff, 2005) and forms the foundation for the paradigm that is employed in the present investigation. In one experiment, for example, subjects began each trial by viewing a display consisting of two square frames, one above and one below a central fixation cross. In the preview display that immediately followed, a letter was presented in each frame. The letters were then removed, and the frames moved continuously to new locations, to the left and right of fixation. When the frames stopped moving, a single target letter was presented within one of the frames, and the subjects' task was to name the target as quickly as possible. Kahneman et al. manipulated the relationship between the preview and the target displays and, in so doing, defined three experimental conditions. In the same-object $(\mathrm{SO})$ condition, the target matched the letter that had been presented within the same frame in the preview display (and was, therefore, part of the same object in both displays; Treisman, Kahneman, \& Burkell, 1983). In the different-object (DO) condition, the target matched the letter that had been present in the opposite frame in the preview display. Finally, in the no-match (NM) condition, the target did not match either of the previewed letters.

On the basis of these three conditions, Kahneman et al. (1992) defined two effects of theoretical interest. The first, which they termed a nonspecific preview benefit, was cal- culated as the difference in reaction time (RT) between the NM and the DO conditions and, therefore, reflected the benefit of previewing the target object, even though it was previewed within the wrong frame. The second effect was termed an object-specific preview benefit and was calculated as the difference in RT between the DO and the $\mathrm{SO}$ conditions. Because subjects received a preview of the target in both conditions, this effect reflects an additional benefit of previewing the target as part of the same perceptual object as that in the target display.

These two effects reflect distinct representational systems that support object priming (Henderson, 1994). Nonspecific effects rely on the representation of object types in long-term memory. These type representations, it is important to note, do not represent specific objects in the environment but general object classes (e.g., different chair tokens within a scene may be represented by a single type representation of chairs). Object-specific effects, on the other hand, rely on representations of specific object tokens that are present at a particular place and time; that is, they rely on object file representations. The presence or absence of object-specific effects is thought to reflect the preservation of object continuity in the SO condition. To understand why this is so, consider how object files are constructed and used during a trial in this experiment. When the preview display is presented, subjects construct new object file representations for the objects above and below fixation. When the frames move to new locations, a correspondence process establishes a link between the objects in the preview display and the objects in the target display. The object files are then retrieved and compared with the target objects. In the SO condition, the target matches its stored representation; in the DO condition, on the other hand, the match fails. As a result, continuity is disrupted; the target is not seen as a continuously present object across the scene change. When asked to name the target objects, subjects do so more slowly in the DO condition than in the SO condition.

Considerable work has been done examining the nature of object file representations (e.g., Gordon \& Irwin, 1996, 2000; Henderson \& Anes, 1994; Henderson \& Siefert, 2001; Mitroff, Scholl, \& Wynn, 2004). In many cases, the approach that researchers have taken is to modify features of the preview and target objects in order to determine which object features play a role in preserving continuity. This issue was not addressed by Kahneman et al. (1992), because in their experiments, when the target and the preview object matched, they matched in all respects. That is, they had the same shape, color, orientation, identity, and so on. Thus, the question remained whether object continuity was supported by continuity of an object's perceptual features or by continuity of its identity. This was addressed in a series of experiments described by Gordon and Irwin (2000), using the same general approach as that used by Kahneman et al. In their Experiment 4, for example, subjects viewed a preview display consisting of two frames, one above and one below fixation. Within each frame was a line drawing of an object. The objects then disappeared, and the frames moved to new locations, to the left and right of fixation. A target was then presented 
in one of the frames. Rather than a picture, however, the target was a word that either named one of the preview objects (in the SO and DO conditions) or named an object that was not in the preview display (in the NM condition). For example, if the preview display contained a picture of a dog within one frame, the target might be the word $d o g$ within one of the frames. The subjects' task was to indicate whether the target was a familiar English word. The results indicated that there was a significant objectspecific benefit, despite the fact that the target shared almost no perceptual features with the preview in either the SO or the DO condition. On the basis of this and similar results, Gordon and Irwin (2000) concluded that object files do not represent perceptual object features. Instead, they argued that object files represent postcategorical information, such as an object's identity.

Recent evidence, however, suggests that this may not always be the case (e.g., Henderson \& Siefert, 2001; Verfaillie \& De Graef, 2000). Henderson and Siefert, for example, examined the transsaccadic representation of object orientation, using a procedure derived from the reviewing paradigm. The subjects in their study began each trial by fixating a marker on the left side of the screen. A preview display then appeared, which consisted of a peripheral fixation marker located between an upper and a lower preview object. One of the preview objects was a line drawing of a familiar object, whereas the other was a meaningless flanker. The subjects were instructed to move their eyes to fixate the peripheral marker as soon as the preview appeared. While the eyes were in motion, the preview was replaced with a target display containing a single familiar object, located above or below the saccade target and accompanied by a meaningless flanker, and the subjects' task was to name the familiar object as quickly as possible. When the target and the preview pictures matched, they were presented in either the same or an opposite location, permitting the calculation of object-specific preview benefits. Importantly, Henderson and Siefert manipulated the orientation of the target, so that it either matched or was a mirror image of the previewed object. The results indicated that there was a significant object-specific benefit when the preview and the target objects were identical but that this benefit was reduced by half when the orientation of the target did not match the orientation of the previewed object. Henderson and Siefert concluded that an object's orientation is part of the episodic representation that underlies transsaccadic memory.

\section{EXPERIMENT 1}

The results of Henderson and Siefert (2001) appear to conflict with Gordon and Irwin's (2000) claim that object files do not represent perceptual object properties. In order to understand these contradictory findings, it may be useful to consider methodological differences between the studies. There are many such differences: Gordon and Irwin (2000), for example, examined representation within a fixation, whereas Henderson and Siefert examined representation across a saccade. A difference that may be particularly important, however, concerns the display timing in the experiments. Whereas Gordon and Irwin (2000) used preview-target stimulus onset asynchronies (SOAs) of between 1.75 and $3 \mathrm{sec}$, Henderson and Siefert, whose preview durations were determined by saccade latency, used much shorter SOAs (less than $250 \mathrm{msec}$ ). This difference in SOA may have a substantial impact on the nature of the representation. As proposed by Kahneman et al. (1992), object files are thought to develop over time, being updated with new information as it becomes available. Because object file representations are dynamic, it is likely that the nature of the representation changes over the range of SOAs used by Gordon and Irwin (2000) and Henderson and Siefert. Although the initial representation may be dominated by an object's perceptual features, the representation may become more abstract over time, with postcategorical information eventually replacing the precise featural information.

Thus, the results of Gordon and Irwin (2000) and Henderson and Siefert (2001) may reflect the evolution of object file representations, with Henderson and Siefert's results driven by an early, perceptual representation, and Gordon and Irwin's (2000) results driven by a later, conceptual representation. We investigated this possibility in Experiment 1 by manipulating preview-target SOA within the transsaccadic preview paradigm employed by Henderson and Siefert.

\section{Method}

Subjects. Thirty undergraduate students at North Dakota State University participated in this study in exchange for course credit. All the subjects had normal or corrected-to-normal vision.

Stimuli. The stimuli consisted of 16 black line-drawn objects (Snodgrass \& Vanderwart, 1980) displayed against a white background. The average size of the stimuli was $2.3^{\circ}$ vertically and $3.1^{\circ}$ horizontally. In addition, on each trial, one of the display locations contained a meaningless placeholder object; Henderson and Siefert (2001) reported that priming effects were enhanced when the preview display contained only 1 meaningful object. The placeholder was made up of two different-sized squares, one set inside of the other, and a fixation cross in the middle of the inner square. The size of the meaningless placeholder was $3.2^{\circ}$ vertically and horizontally. Six of the line-drawn objects (ant, bird, frog, horse, mouse, and shoe) were used only for practice trials. The remaining 10 linedrawn objects (camel, cow, dog, elephant, fish, flag, football, iron, squirrel, and whistle) were used in the experimental trials. These same 10 objects were used by Henderson and Siefert.

Apparatus. The stimuli were presented at a resolution of 1,024 $\times$ 768 pixels on an NEC MultiSync FP2141SB color monitor, with a refresh rate of $75 \mathrm{~Hz}$. The subjects viewed the screen from a distance of $57 \mathrm{~cm}$, with a chinrest to reduce head movement. At this distance, the size of the total display area was $32^{\circ}$ vertically and $45^{\circ}$ horizontally. Both the fixation cross located in the center of the screen and the saccade target had a height and width of $0.6^{\circ}$. The distance from the fixation cross to the saccade target was $16.4^{\circ}$. The distance from the saccade target to the center of each stimulus was $4.7^{\circ}$. The subjects' eye movements were recorded using a head-mounted EyeLink II eyetracker (SR Research Ltd., Mississauga, ON, Canada) configured to sample eye position at $250 \mathrm{~Hz}$. The eyetracker was calibrated at the beginning of the experiment, and a drift correction was performed at the start of each trial. The subjects responded by speaking into a microphone attached to a voice key (Cedrus SV-1 Smart Voice Key, San Pedro, CA) to provide naming latencies.

Procedure. The subjects completed two experimental blocks, which differed with respect to SOA. In the immediate move block, the subjects initiated the trial sequence by focusing on a fixation 
cross on the left side of the screen and pressing the "Enter" key on the keyboard. Following a 1.5 -sec interval, two line-drawn objects, one of which was always a meaningless flanker, appeared on the right side of the screen, above and below a saccade target (which was identical to the fixation cross). The meaningful preview object appeared equally often in the top and bottom preview locations. The preview display remained on the screen until a saccade was initiated A saccade was detected when eye velocity exceeded $30 \%$ sec or eye acceleration exceeded $9,500^{\circ} / \mathrm{sec}^{2}$. Across subjects, the mean saccade latency was $240 \mathrm{msec}(S D=68.7 \mathrm{msec})$.

In the delayed move block, the subjects initiated the trial sequence as in the previous block; however, in this block, the subjects were required to wait for a $240-\mathrm{msec}, 400-\mathrm{Hz}$ tone to cue them to move their eyes to the saccade target. When the subjects initiated the trial, the preview display was presented as in the immediate move condition. The duration of the preview display was equal to each subject's average saccade latency in the immediate move condition. The objects were then removed from the screen, and $1.5 \mathrm{sec}$ later, the auditory cue was presented. The subjects were instructed to move their eyes to the saccade target when the cue was presented.

During the saccade, the preview display was replaced with one of three target displays, corresponding to the three target conditions described by Kahneman et al. (1992). In the SO condition, the target object matched the object presented in the same location in the pre- vious display. In the DO condition, the target matched the previewed object but appeared in the opposite location. In the final condition, the NM condition, the object in the target display did not match the previewed object. The orientation of the target object was manipulated as well. When the target appeared in the SO or DO condition, it appeared in either the same or a different orientation as in the preview. In the different-orientation condition, the target was a mirror image of the previewed object. An example of the display conditions used in Experiment 1 is provided in Figure 1.

The subjects responded by naming the target object. RT was measured from the onset of the target object until the voice key was triggered. After the subjects named the target, the experimenter noted whether or not the subjects had given the correct name for the object. To control for voice key triggering time by different phonemes, we scored a name as correct only when it matched the desired name exactly; for example, rat would not be an accepted label for the target object mouse (even though it accurately describes the object) and, therefore, would be counted as an error and excluded from the analyses. In addition, trials on which the subjects spoke too softly to trigger the voice key or triggered the voice key prematurely by making unrelated noise were excluded from the analyses, although not counted as errors. The experiment lasted approximately $40 \mathrm{~min}$. Each subject completed two blocks of 120 experimental trials, with 12 practice trials preceding each block. Within each block, condi-

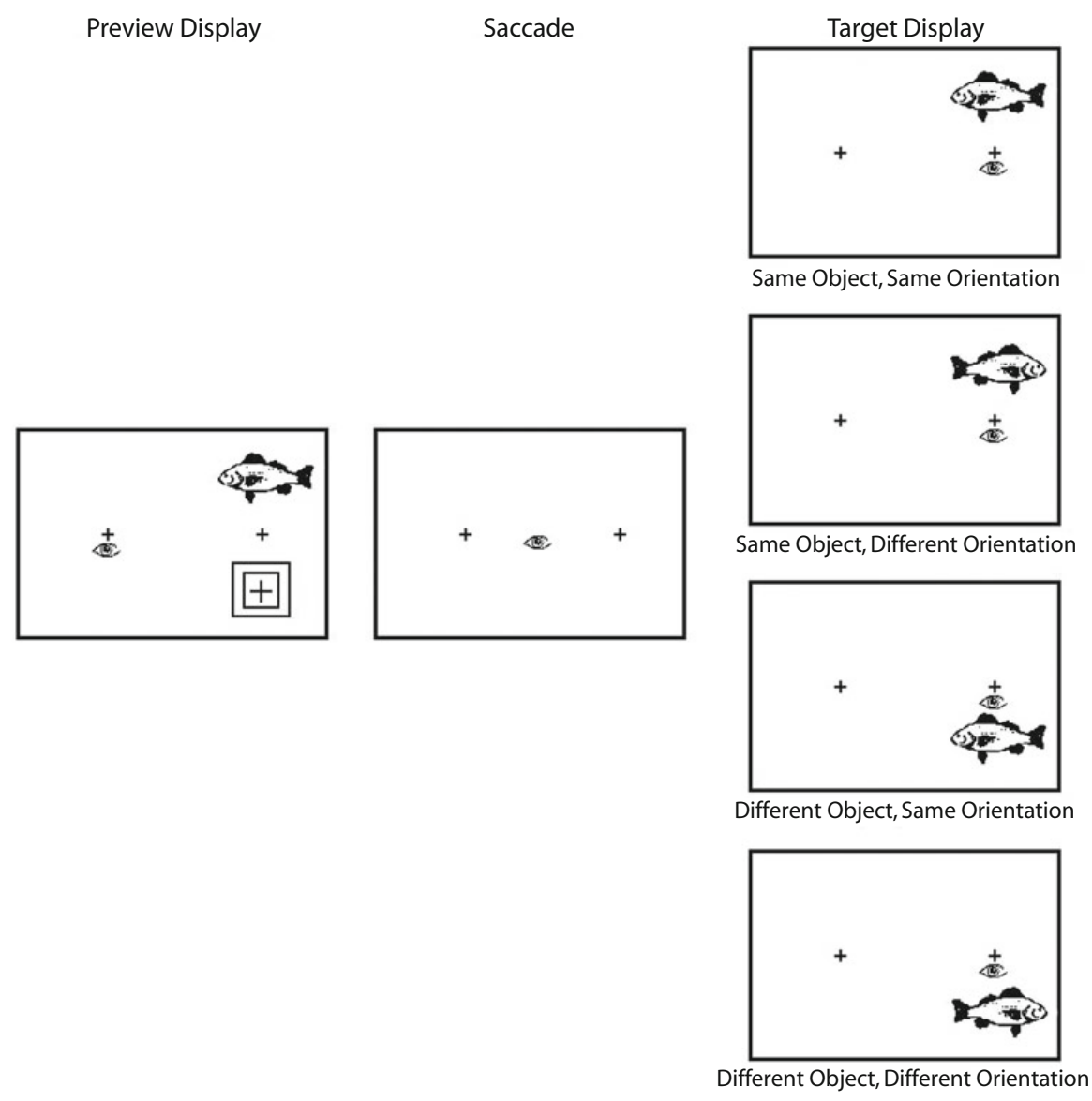

Figure 1. Example of the Experiment 1 procedure. The subject's task was to name the object in the target display. In the no-match condition (not shown), the target object's identity did not match the identity of the object in the preview display. 
tions were interleaved. Each object appeared as a target 12 times within a trial block.

The subjects were instructed to move their eyes to the saccade target located between the preview stimuli. To assess compliance with these instructions, we analyzed saccade landing locations for each trial. Following the example of Gajewski and Henderson (2005), we defined a saccade target region that extended $3^{\circ}$ above and below the saccade target and excluded the preview objects; saccades were considered to be on target if they landed within this region. The results of this analysis revealed substantial variability in saccade accuracy, with some subjects fixating the saccade target on $92.9 \%$ of the trials and others fixating the saccade target on just $34.6 \%$ of the trials. Overall, saccades were made to the saccade target on $62.9 \%$ of the trials. Although this represents a smaller proportion of on-target saccades than that reported by Gajewski and Henderson $(M=84 \%)$, our subjects were much more likely to fixate the saccade target than were the subjects in a similar study by Germeys et al. (2002; $M=$ $3 \%$ ). Off-target saccades were more likely to select the bottom object $(42.6 \%$ of the trials) than to select the top object (31.6\% of the trials) and were more likely at the short SOA (48.8\% of the trials) than at the long SOA ( $25.4 \%$ of the trials). This difference likely reflects the fact that the preview objects were no longer visible when the saccade was executed at the long SOA.

Gajewski and Henderson (2005) conducted a careful analysis of the relationship between object-specific and nonspecific preview benefits and saccade landing position and found that neither benefit was affected by errors in saccade targeting. Nonetheless, we conducted an additional set of analyses in which trials containing off-target saccades were excluded. In addition, 11 subjects were excluded from this analysis because they made off-target saccades on more than $40 \%$ of the trials. The results of this additional analysis will be reported below. As these results make clear, excluding trials containing off-target saccades did not substantially change the pattern of results in Experiment 1.

\section{Results}

Before RTs were analyzed, the data were trimmed by eliminating RTs greater than $2 \mathrm{sec}$ or those that differed by more than 2.5 standard deviations from that subject's mean RT for that condition ( $\mathrm{SO}, \mathrm{DO}$, or $\mathrm{NM})$ and orientation (same or different). These criteria eliminated $3.6 \%$ of the trials from analysis. We also eliminated trials containing anticipatory saccades (defined as saccades with latencies less than $100 \mathrm{msec}$ ); this criterion eliminated an additional $3.9 \%$ of the trials from the analysis. The data were then analyzed in a 3 (condition) $\times 2$ (orientation) $\times 2(\mathrm{SOA})$ ANOVA. Two sets of analyses are reported below. The first, subscripted "1," includes all trials, regardless of the accuracy of the initial saccade; the second, subscripted " 2 ," excludes trials on which the initial saccade was not made to the saccade target. Where means are provided below, they refer to the analysis in which all the trials are included.

Mean RTs and error rates for each condition are reported in Table 1 for both sets of analyses. In the analyses that follow, all effects are significant at the .05 level, except as otherwise noted. The results of an ANOVA performed on the RTs revealed a main effect of SOA, with longer RTs following short SOAs $(M=816 \mathrm{msec})$ than following long SOAs $(M=790 \mathrm{msec})\left[F_{1}(1,29)=6.1\right.$, $\left.M S_{\mathrm{e}}=9,766 ; F_{2}(1,18)=4.5, M S_{\mathrm{e}}=13,031\right]$. There was also a main effect of orientation, since RTs were shorter when the orientation of the target matched its preview orientation $(M=791 \mathrm{msec})$ than when it did not $(M=$ $814 \mathrm{msec})\left[F_{1}(1,29)=29.2, M S_{\mathrm{e}}=1,620 ; F_{2}(1,18)=\right.$ $\left.75.4, M S_{\mathrm{e}}=3,229\right]$. There was also a main effect of condition $\left[F_{1}(2,58)=152.0, M S_{\mathrm{e}}=1,828 ; F_{2}(2,36)=22.3\right.$, $\left.M S_{\mathrm{e}}=2,783\right]$; the subjects were slower to name objects in the DO condition $(M=807 \mathrm{msec})$ than in the SO condition $(M=753 \mathrm{msec})$ and were slower still in the NM condition $(M=849 \mathrm{msec})$. These differences are consistent with the predicted object-specific and nonspecific preview benefits. The object-specific effects will be examined below; planned comparisons confirmed that there was significant nonspecific priming $\left[F_{1}(1,58)=14.2\right.$, $\left.M S_{\mathrm{e}}=1,828 ; F_{2}(1,36)=5.0, M S_{\mathrm{e}}=2,783\right]$.

The effect of condition interacted with the effect of SOA $\left[F_{1}(2,58)=24.9, M S_{\mathrm{e}}=2,390 ; F_{2}(2,36)=4.8\right.$, $\left.M S_{\mathrm{e}}=4,458\right]$, with both the object-specific and the nonspecific preview benefits appearing to diminish over time. There was also a significant interaction between SOA and orientation $\left[F_{1}(1,29)=6.5, M S_{\mathrm{e}}=1,999 ; F_{2}(1,18)=\right.$ $\left.8.9, M S_{\mathrm{e}}=3,965\right]$; this reflects the fact that the effect of orientation change was larger following a short SOA $(35 \mathrm{msec})$ than following a long SOA $(11 \mathrm{msec})$. There was no orientation $\times$ condition interaction or three-way interaction (both $F_{\mathrm{S}}<1$ ).

Of primary theoretical interest were the object-specific preview effects and the interaction of those effects with SOA and orientation change. To assess these effects and interactions, an additional ANOVA was conducted that excluded the NM trials (thus permitting an examination of object-specific effects). This analysis confirmed that there was a significant object-specific effect $\left[F_{1}(1,29)=139.4\right.$, $\left.M S_{\mathrm{e}}=1,274 ; F_{2}(1,18)=4.8, M S_{\mathrm{e}}=2,519\right]$. The effect interacted with SOA in the full analysis $\left[F_{1}(1,29)=35.3\right.$, $\left.M S_{\mathrm{e}}=1,697\right]$, but not in the set of analyses in which trials with off-target saccades were excluded $\left[F_{2}(1,18)<1\right]$. The nature of this interaction in the full analysis was that the object-specific effect was larger following a short SOA $(M=86 \mathrm{msec})$ than following a long SOA $(M=$ $23 \mathrm{msec}$ ). Planned comparisons confirmed, however, that the object-specific effect was significant at both the short

Table 1

Mean Reaction Times (RTs, in Milliseconds) and Error Rates (ERs, in Percentages) in Experiment 1, With Preview Effects

\begin{tabular}{|c|c|c|c|c|}
\hline \multirow[b]{3}{*}{ Condition } & \multicolumn{4}{|c|}{ Orientation } \\
\hline & \multicolumn{2}{|c|}{ Same } & \multicolumn{2}{|c|}{ Different } \\
\hline & RT & ER & RT & ER \\
\hline \multicolumn{5}{|c|}{ Short SOA } \\
\hline Same object & 725 & 0.7 & 756 & 0.4 \\
\hline Different object & 813 & 0.9 & 840 & 0.9 \\
\hline No match & 856 & 3.8 & 903 & 5.0 \\
\hline \multicolumn{5}{|l|}{ Preview Effects } \\
\hline Object-specific benefit & 88 & 0.2 & 84 & 0.5 \\
\hline Nonspecific benefit & 43 & 2.9 & 63 & 4.1 \\
\hline \multicolumn{5}{|c|}{ Long SOA } \\
\hline Same object & 761 & 1.0 & 769 & 1.2 \\
\hline Different object & 783 & 1.3 & 791 & 1.1 \\
\hline No match & 809 & 1.3 & 826 & 1.6 \\
\hline \multicolumn{5}{|l|}{ Preview Effects } \\
\hline Object-specific benefit & 22 & 0.3 & 22 & -0.1 \\
\hline Nonspecific benefit & 26 & 0.0 & 35 & 0.5 \\
\hline
\end{tabular}

Note-SOA, stimulus onset asynchrony. 
SOA $\left[F_{1}(1,29)=65.4, M S_{\mathrm{e}}=1,697\right]$ and the long SOA $\left[F_{1}(1,29)=4.6, M S_{\mathrm{e}}=1,697\right]$. Contrary to the results of Henderson and Siefert (2001), the object-specific effect was not affected by a change in orientation; the $95 \%$ confidence interval for this interaction was $5 \pm 22 \mathrm{msec}$, suggesting that there was sufficient power to detect an interaction of the size reported by Henderson and Siefert. There was no three-way interaction of the effect with SOA and orientation change (all $F_{\mathrm{S}}<1$ ).

Analyses of the error data were generally consistent with those of the RT data and did not suggest a speedaccuracy trade-off (see Table 1). There was a significant effect of condition, with error rates of $0.8 \%, 1.1 \%$, and $2.9 \%$ in the $\mathrm{SO}, \mathrm{DO}$, and NM conditions, respectively $\left[F_{1}(2,58)=11.9, M S_{\mathrm{e}}=13.3 ; F_{2}(2,36)=3.7, M S_{\mathrm{e}}=\right.$ $1.8]$. There was no effect of orientation $\left[F_{1}(1,29)<1\right]$, although the effect was nearly significant in the analysis of on-target trials $\left[F_{2}(1,18)=3.6, M S_{\mathrm{e}}=5.1, p<.05\right]$. As was the case for the RT data, there was a condition $\times$ SOA interaction in the full analysis $\left[F_{1}(2,58)=8.0, M S_{\mathrm{e}}=\right.$ 14.6], but not in the analysis that excluded off-target trials $\left[F_{2}(2,36)<1\right]$. There was no condition $\times$ orientation interaction $\left[F_{1}(2,58)=2.5, M S_{\mathrm{e}}=3.1 ; F_{2}(2,36)=1.5\right.$, $\left.M S_{\mathrm{e}}=3.1\right]$. No other main effects or interactions were significant in the full analysis (all $F \mathrm{~s}<1$ ); in the analysis of on-target trials, there was a significant SOA $\times$ orientation interaction $\left[F_{2}(1,18)=5.1, M S_{\mathrm{e}}=6.2\right]$. Planned comparisons revealed no significant object-specific effects (both $F \mathbf{s}<1$ ) or nonspecific effects $\left[F_{1}(1,58)=3.7\right.$, $\left.M S_{\mathrm{e}}=13.3, p>.05 ; F_{2}(1,36)<1\right]$.

\section{Discussion}

The goal of Experiment 1 was to investigate changes in object file representation over time, by manipulating preview-target SOA in a transsaccadic priming paradigm. Increasing SOA dramatically affected the size of the object-specific preview benefit, reducing it by nearly two thirds. This reduction may suggest that object file representations decay rather rapidly in the absence of a continuously visible object, although it should be emphasized that the object-specific effect, although smaller, was nevertheless reliable even at the long SOA. Given that Noles et al. (2005) found that object files can persist as long as $8 \mathrm{sec}$ when an object frame provides spatiotemporal continuity during the interstimulus period, it may be that surrounding the objects in the present experiment with frames that remained visible throughout the trial reduced the effect of SOA.

Although the results suggest that object file representations decay over time, they do not suggest that the nature of the representation changes during that time. At both the short and the long SOAs, the data suggest that object continuity is dependent on continuity of abstract properties, rather than physical properties, such as orientation. Surprisingly, therefore, the results of Experiment 1 failed to confirm Henderson and Siefert's (2001) finding that object-specific preview benefits are reduced when the orientation of the target is different than its preview orientation. However, the present findings are consistent with previous research (e.g., Gordon \& Irwin, 2000; Hender- son, 1994) showing that changes in an object's physical form do not reduce or eliminate object-specific priming. One possible source of these conflicting results was assessed in Experiment 2.

\section{EXPERIMENT 2}

We believe that properties of the object-priming task are likely to influence the extent to which orientation (or any perceptual property) plays a role in preserving object continuity. Although we replicated most of the conditions under which Henderson and Siefert (2001) obtained their results, there were key differences between our study and theirs. A difference that may have had profound consequences for our results concerns the eccentricity of the preview stimuli. In Henderson and Siefert's study, preview objects were presented $10^{\circ}$ from fixation, whereas the eccentricity in Experiment 1 was $16.4^{\circ}$. Given the degradation in image quality at this greater eccentricity, it is possible that Henderson and Siefert's subjects were better able to form and maintain a detailed perceptual representation and that this representation was more likely to influence the comparison process. In Experiment 2, we replicated the design of our Experiment 1, using preview displays that were closer to fixation.

\section{Method}

Subjects. Eighteen undergraduate students at North Dakota State University participated in this study in exchange for course credit. All the subjects had normal or corrected-to-normal vision.

Stimuli. The stimuli were identical to those used in Experiment 1, with the exception that the distance between the central fixation cross and the saccade target located between the preview stimuli was $11^{\circ}$ (comparable to the $10^{\circ}$ eccentricity used by Henderson \& Siefert, 2001), rather than $16.4^{\circ}$.

Apparatus. The apparatus was identical to that used in Experiment 1 .

Procedure. The procedure was identical to that used in Experiment 1. As in Experiment 1, SOA was blocked, with subjects completing a block of immediate move trials prior to completing a block of delayed move trials. The mean saccade latency, which determined the preview display duration for each subject, was $204 \mathrm{msec}(S D=$ $45.5 \mathrm{msec})$.

\section{Results}

Before RTs were analyzed, the data were trimmed by eliminating RTs greater than $2 \mathrm{sec}$ or those that differed by more than 2.5 standard deviations from that subject's mean RT for that condition (SO, DO, or NM) and orientation (same or different). These criteria eliminated 1.2\% of the trials from analysis. We also eliminated trials containing anticipatory saccades (defined as saccades with latencies less than $100 \mathrm{msec}$ ); this criterion eliminated an additional $5.0 \%$ of the trials from the analysis. The data were then analyzed in a 3 (condition) $\times 2$ (orientation) $\times$ 2 (SOA) ANOVA. As in Experiment 1, we conducted two sets of analyses: one (subscripted " 1 ") in which all trials were included, and another (subscripted " 2 ") that excluded the $31 \%$ of trials on which the initial saccade was not made to the saccade target.

Mean RTs and error rates for each condition are reported in Table 2. The results of an ANOVA performed on 
Table 2

Mean Reaction Times (RTs, in Milliseconds) and Error Rates (ERs, in Percentages) in Experiment 2, With Preview Effects

\begin{tabular}{|c|c|c|c|c|}
\hline \multirow[b]{3}{*}{ Condition } & \multicolumn{4}{|c|}{ Orientation } \\
\hline & \multicolumn{2}{|c|}{ Same } & \multicolumn{2}{|c|}{ Different } \\
\hline & RT & ER & RT & ER \\
\hline \multicolumn{5}{|c|}{ Short SOA } \\
\hline Same object & 723 & 0.8 & 733 & 1.7 \\
\hline Different object & 851 & 1.1 & 799 & 4.2 \\
\hline No match & 826 & 1.1 & 887 & 4.2 \\
\hline \multicolumn{5}{|l|}{ Preview Effects } \\
\hline Object-specific benefit & 128 & 0.3 & 66 & 2.5 \\
\hline Nonspecific benefit & -25 & 0.0 & 88 & 0.0 \\
\hline \multicolumn{5}{|c|}{ Long SOA } \\
\hline Same object & 770 & 1.1 & 797 & 1.1 \\
\hline Different object & 799 & 0.6 & 801 & 1.9 \\
\hline No match & 848 & 1.7 & 821 & 0.8 \\
\hline \multicolumn{5}{|l|}{ Preview Effects } \\
\hline Object-specific benefit & 29 & -0.5 & 4 & 0.8 \\
\hline Nonspecific benefit & 49 & 1.1 & 20 & -1.1 \\
\hline
\end{tabular}

Note-SOA, stimulus onset asynchrony.

the RTs revealed a main effect of orientation $\left[F_{1}(1,17)=\right.$ 7.1, $\left.M S_{\mathrm{e}}=1,683 ; F_{2}(1,17)=4.5, M S_{\mathrm{e}}=3,130\right]$, with longer RTs when the orientation changed $(M=803 \mathrm{msec})$ than when it did not $(M=788 \mathrm{msec})$. There was no effect of SOA (both $F \mathrm{~s}<1$ ); there was, however, a main effect of condition, with mean RTs of 745,790 , and $853 \mathrm{msec}$ in the SO, DO, and NM conditions, respectively $\left[F_{1}(2,34)=63.7, M S_{\mathrm{e}}=3,281 ; F_{2}(2,34)=32.3, M S_{\mathrm{e}}=\right.$ $5,288]$. This effect interacted with SOA $\left[F_{1}(2,34)=22.3\right.$, $\left.M S_{\mathrm{e}}=3,287 ; F_{2}(2,34)=12.2, M S_{\mathrm{e}}=4,160\right]$, since both the object-specific and the nonspecific preview effects appeared to diminish at longer SOAs (see below). There was also a significant condition $\times$ orientation interaction $\left[F_{1}(2,34)=8.7, M S_{\mathrm{e}}=1,140 ; F_{2}(2,34)=7.3, M S_{\mathrm{e}}=\right.$ $1,959]$; the theoretical significance of this interaction will be discussed below. There was also a significant orientation $\times$ SOA interaction $\left[F_{1}(1,17)=5.6, M S_{\mathrm{e}}=2,070\right.$; $\left.F_{2}(1,17)=5.9, M S_{\mathrm{e}}=2,014\right]$. The three-way interaction of orientation, condition, and SOA failed to reach significance $\left[F_{1}(2,34)=2.9, M S_{\mathrm{e}}=2,220 ; F_{2}(2,34)=2.5\right.$, $M S_{\mathrm{e}}=3,422$; both $\left.p \mathrm{~s}>.05\right]$.

As in Experiment 1, we conducted an additional ANOVA that excluded trials in the NM condition, in order to focus on the object-specific effects that are of primary theoretical importance. This analysis confirmed that there was a significant object-specific effect $\left[F_{1}(1,17)=18.6, M S_{\mathrm{e}}=\right.$ 3,$\left.766 ; F_{2}(1,17)=14.2, M S_{\mathrm{e}}=4,755\right]$ that interacted with SOA $\left[F_{1}(1,17)=30.0, M S_{\mathrm{e}}=1,993 ; F_{2}(1,17)=20.3\right.$, $\left.M S_{\mathrm{e}}=2,586\right]$. Importantly, the effect also interacted with orientation $\left[F_{1}(1,17)=4.8, M S_{\mathrm{e}}=1,202\right]$, although the interaction was only marginally significant in the analysis of on-target trials $\left[F_{2}(1,17)=4.0, M S_{\mathrm{e}}=1,717, p=.06\right]$; the object-specific effect was larger when the orientation of the target matched its preview orientation $(57 \mathrm{msec})$ than when it did not $(31 \mathrm{msec})$. These results thus confirm Henderson and Siefert's (2001) findings that orientation changes disrupt perceptual continuity, for objects located within approximately $10^{\circ}$ of fixation.
Analyses of the error data revealed main effects of condition $\left[F_{1}(2,34)=3.3, M S_{\mathrm{e}}=4.2 ; F_{2}(2,34)=2.5, M S_{\mathrm{e}}=\right.$ $3.0, p<.10]$ and orientation $\left[F_{1}(1,17)=7.6, M S_{\mathrm{e}}=11.1\right.$; $\left.F_{2}(1,17)=7.5, M S_{\mathrm{e}}=13.0\right]$, as well as an effect of orientation that narrowly missed significance in the full analysis $\left[F_{1}(1,17)=4.4, M S_{\mathrm{e}}=11.6 ; F_{2}(1,17)<1\right]$. There was a significant SOA $\times$ orientation interaction $\left[F_{1}(1,17)=\right.$ $\left.7.2, M S_{\mathrm{e}}=8.5 ; F_{2}(1,17)=6.7, M S_{\mathrm{e}}=5.0\right]$, but there were no SOA $\times$ condition $\left[F_{1}(2,34)=1.6, M S_{\mathrm{e}}=5.9\right.$; $\left.F_{2}(2,34)=2.6, M S_{\mathrm{e}}=3.4\right]$, orientation $\times$ condition $\left[F_{1}(2,34)=2.4, M S_{\mathrm{e}}=6.4 ; F_{2}(2,34)=1.6, M S_{\mathrm{e}}=5.4\right]$, or three-way $\left[F_{1}(2,34)=2.7, M S_{\mathrm{e}}=4.1 ; F_{2}(2,34)=2.3\right.$, $\left.M S_{\mathrm{e}}=3.9\right]$ interactions. Planned comparisons revealed no significant nonspecific effect (both $F_{\mathrm{s}}<1$ ). There was, however, a significant object-specific effect, which was explored in an ANOVA that excluded trials from the NM condition $\left[F_{1}(1,17)=8.0, M S_{\mathrm{e}}=2.6 ; F_{2}(1,17)=5.8\right.$, $\left.M S_{\mathrm{e}}=1.9\right]$. As in the analysis of the RT data, the ANOVA revealed a significant interaction of orientation and condition $\left[F_{1}(1,17)=8.1, M S_{\mathrm{e}}=3.6 ; F_{2}(1,17)=11.1, M S_{\mathrm{e}}=\right.$ 1.5]. Surprisingly, this interaction reflects a slightly larger object-specific effect when the orientation of the target changed $(1.7 \%)$ than when it stayed the same $(-0.1 \%)$.

\section{Discussion}

The goal of Experiment 2 was to investigate a failure in Experiment 1 to replicate Henderson and Siefert's (2001) finding that changes in object orientation disrupt perceptual continuity, reducing object-specific preview effects. Although the design of Experiment 1 followed that of their Experiment 2 quite closely, objects were presented much further from fixation in our experiment than in theirs. Given the reductions in image quality that accompany increases in retinal eccentricity, the large eccentricity used in Experiment 1 may have reduced the quality of the perceptual representation of the preview objects, making it less likely that changes in orientation would threaten perceptual stability. Thus, in Experiment 2, we reduced the eccentricity of the preview display, more closely replicating the conditions in Henderson and Siefert's study.

As was predicted, we were able to replicate their key finding, lending additional support to their conclusion that changes in orientation disrupt an object's stability across a saccade. The results of Experiment 1 suggest, however, that the extent to which such changes disrupt stability depends on the eccentricity at which the object is viewed prior to the saccade. More generally, we argue that the quality of a perceptual representation determines the likelihood that changes in its properties will disrupt stability. Representations of objects seen only in far peripheral vision, as in Experiment 1, may be robust to changes in orientation by virtue of the fact that the objects' orientation is poorly represented to begin with.

Indeed, it may be the case that the impoverished input available from objects far from fixation restricts an observer's ability to form stable representations of those objects. Such an interpretation, although consistent with the results of Experiment 1, would hardly be revealing of the principles underlying episodic object representation. Arguing against this interpretation is the finding in Ex- 
periment 1 of a main effect of orientation change, which suggests that subjects are able to acquire information about the orientation of peripheral objects. However, this finding must be interpreted cautiously, for two reasons. First, the finding suggests only that object orientation is acquired, but not that it becomes part of a stable representation, such as an object file. Second, the main effect of orientation was not replicated in Experiment 2, when NM trials (for which orientation condition was meaningless) were excluded from the analysis. Furthermore, Henderson and Siefert (2001) found evidence that orientation change disrupts object continuity but did not report a main effect of orientation change. Thus, a main effect of orientation change in Experiment 1 does not appear to provide compelling evidence for the construction of a stable representation that might support object continuity. In Experiment 3, we explicitly assessed representations of object orientation in order to confirm that subjects are able to form stable representations of the orientation of peripheral objects.

Even if subjects are able to form object-specific representations of object orientation, those representations may play a reduced role in preserving object continuity when they are acquired from objects in the periphery. This may reflect a more general bias against the use of unreliable information in perceptual processing (e.g., Atkins, Fiser, \& Jacobs, 2001). This alternative explanation was tested in Experiment 3.

\section{EXPERIMENT 3}

We carried out an additional experiment in order to further examine the acquisition and representation of orientation and the role of orientation information in preserving object continuity. On each trial, we presented preview objects at the same eccentricity as that in Experiment 1 and asked the subjects to explicitly report the orientation of the preview objects within the reviewing paradigm. If the orientation of distant preview objects is not acquired or does not become part of a stable episodic object representation, we would expect the subjects to perform quite poorly on this task. If, however, the results of Experiment 1 suggest only that orientation plays a reduced role in preserving object continuity as eccentricity increases, we would expect the subjects to perform well on this task, even while replicating the finding from Experiment 1 that orientation change does not disrupt continuity.

In addition, we sought to extend the results of Experiment 1 to a different class of objects. Specifically, we replaced the line-drawn objects in Experiment 1 with a set of colored 3-D objects. The new stimuli offered several advantages. In addition to providing increased realism in their depictions of familiar objects, they enabled us to examine object continuity following rotations in depth. This is important, because the manipulation of orientation in Experiments 1 and 2-mirror reversal-may represent a special class of orientation change (e.g., Cooper, Schacter, Ballesteros, \& Moore, 1992) whose impact may differ from that of orientation change more generally. In Experi- ment 3, we examined the robustness of object representations with a broader range of rotation changes.

\section{Method}

Subjects. Sixteen undergraduate students at North Dakota State University participated in exchange for course credit. All the subjects had normal or corrected-to-normal vision.

Stimuli. The stimuli consisted of 10 colored, 3-D objects (provided by Michael J. Tarr, Brown University, Providence, RI). Each of the 10 stimuli (banana, clock, umbrella, fan, scissors, spoon, fork, bed, stapler, and chair) was seen at two viewing angles, with $60^{\circ}$ between each view. The average size of the stimuli was $4.1^{\circ}$ vertically and $4.0^{\circ}$ horizontally.

Apparatus. The apparatus was the same as that in Experiment 1, except that the distance from the saccade target to the center of each stimulus was $5.3^{\circ}$. In the second set of analyses reported below, we excluded trials on which the initial saccade landed outside of a region that extended $2.5^{\circ}$ above and below the saccade target.

Procedure. The procedure was very similar to that used in the immediate move condition in Experiment 1. In Experiment 3, the subjects initiated the trial sequence by fixating a small cross in the center of the screen and then pressing the space bar. Two objects then appeared, one above the other, with a saccade target located midway between them on the right side of the viewing screen. Unlike in Experiment 1, both objects were meaningful and familiar objects. Although using two meaningful preview objects carried the risk of reducing the overall object-specific effect (e.g., Henderson \& Anes, 1994), it was necessary in order to ensure that the subjects were required to rely on an object-specific representation to perform the orientation judgment task. Had the preview display contained only a single object, for example, it may have been sufficient for the subjects to encode orientation information (such as the word left) without tying that representation to a particular object within the display.

When the preview display appeared, the subjects shifted fixation to the saccade target. Objects remained on the screen until a saccade was detected, which occurred when the eyes crossed an invisible boundary between the fixation cross and the saccade target. The location of the boundary was $7.7^{\circ}$ from the fixation cross and $8.7^{\circ}$ from the saccade target. The eccentricity of the saccade target was $16.4^{\circ}$, as in Experiment 1. On average, the subjects moved their eyes to the target with a saccade latency of $556 \mathrm{msec}(S D=340.6 \mathrm{msec})$. This is considerably longer than the saccade latency in Experiment 1 $[t(44)=4.95, p<.05]$, suggesting that the presence of the secondary orientation judgment task may have encouraged the subjects to delay saccades in order to encode target object orientations. Alternatively, this result may reflect the addition of a second preview object, although Henderson and his colleagues (e.g., Henderson, 1994; Henderson \& Anes, 1994) have not previously reported such dramatic increases in saccade latency with the addition of a second preview object.

During the saccade, the preview display was replaced with a target display. The target object appeared either in the same location as the preview (the SO condition) or in the location of the other meaningful object (the DO condition). The subjects responded by naming the target object. RT was measured from the onset of the target object until the voice key was triggered. Unlike in Experiment 1, there was no NM condition in Experiment 3, because the object-specific preview effect was of greatest theoretical interest and because it would not have been possible for the subjects to carry out the orientation judgment task (described below) on NM trials.

After naming the target, the subjects completed an orientation judgment task, in which they indicated whether the target's orientation matched its orientation in the preview display. The target display remained visible while the subjects completed the orientation judgment task. The subjects responded by clicking one of two mouse buttons. After the subjects had completed the orientation judgment task, the experimenter recorded whether or not the subjects had given the 
correct name for the target. As in Experiment 1, we scored a name as correct only when it matched the desired name exactly. The subjects were not given the correct names in advance; however, pilot data indicated general naming agreement among naive subjects shown the target objects.

The subjects completed one block of 12 practice trials and one block of 80 experimental trials. Across subjects, each target object appeared in every location (top or bottom), condition (same object or different object), and orientation. The duration of the experiment was approximately $30 \mathrm{~min}$.

\section{Results}

Before RTs were analyzed, the data were trimmed by eliminating RTs greater than $2 \mathrm{sec}$ or those that differed by more than 2.5 standard deviations from that subject's mean RT for that condition (SO or DO) and orientation (same or different). These criteria eliminated $3.7 \%$ of the trials from analysis. We also eliminated trials containing anticipatory saccades; this criterion eliminated $2.4 \%$ of the trials from the analysis. The data were then analyzed in a 2 (condition) $\times 2$ (orientation) ANOVA. As in Experiments 1 and 2, we conducted two sets of analyses: one (subscripted " 1 ") in which all the trials were included, and another (subscripted " 2 ") that excluded the $24.1 \%$ of the trials on which the initial saccade was not made to the saccade target. The second set of analyses also excluded 4 subjects who made off-target saccades on more than $40 \%$ of the trials.

Mean RTs and error rates for each condition are reported in Table 3. An ANOVA performed on the RT data revealed no effect of orientation $\left[F_{1}(1,15)=1.1, M S_{\mathrm{e}}=2,160\right.$; $\left.F_{2}(1,11)<1\right]$. Importantly, however, there was a significant object-specific effect $\left[F_{1}(1,15)=5.1, M S_{\mathrm{e}}=2,855\right]$, with the subjects responding more quickly in the SO condition $(M=1,025 \mathrm{msec})$ than in the DO condition $(M=$ $1,055 \mathrm{msec})$. There was insufficient power to confirm this effect in the reduced analysis $\left[F_{2}(1,11)=1.8, M S_{\mathrm{e}}=7,979\right]$, although the size of the object-specific effect $(35 \mathrm{msec})$ was comparable to that in the full analysis $(30 \mathrm{msec})$. As in Experiment 1 , the object-specific effect did not interact with orientation (both $F_{\mathrm{s}}<1$ ), since the effect was as large in the same-orientation condition ( $32 \mathrm{msec})$ as in the differentorientation condition $(29 \mathrm{msec})$; the $95 \%$ confidence interval for testing this interaction was $3 \pm 22 \mathrm{msec}$. In both Experiments 1 and 3, therefore, the results suggest that an object's orientation does not play a significant role in preserving its continuity across a saccade, when the eccentricity at which the object is previewed is large.

If this reflected a failure to represent an object's orientation across a saccade, subjects should have performed at

Table 3

Mean Reaction Times (RTs, in Milliseconds) and Error Rates (ERs, in Percentages) in Experiment 3, With Preview Effects

\begin{tabular}{lccccc}
\hline & \multicolumn{4}{c}{ Orientation } \\
\cline { 2 - 3 } \cline { 5 - 6 } \multicolumn{1}{c}{ Condition } & \multicolumn{2}{c}{ Same } & & \multicolumn{2}{c}{ Different } \\
\cline { 2 - 3 } \cline { 5 - 6 } & RT & ER & & RT & ER \\
\hline Same object & 1,018 & 0.9 & & 1,032 & 0.3 \\
Different object & 1,050 & 0.9 & & 1,061 & 0.9 \\
$\begin{array}{c}\text { Preview Effects } \\
\text { Object-specific benefit }\end{array}$ & 32 & 0.0 & & 29 & 0.6 \\
\hline
\end{tabular}

chance on the orientation judgment task, in which they were asked to indicate whether the target's orientation matched its orientation in the preview display. However, the data from that task suggest that the subjects represented orientation information very well: They performed well above chance $[t(15)=12.3]$, with a mean accuracy of $78.4 \%$. Thus, the results suggest that the insensitivity of object-specific priming to changes in orientation is not due to a failure to maintain an object-specific representation of orientation.

An ANOVA performed on the error data revealed no main effect of orientation or condition (all $F \mathrm{~s}<1$ ) and no interaction between the two variables $\left[F_{1}(1,15)=2.1\right.$, $\left.M S_{\mathrm{e}}=0.73 ; F_{2}(1,11)=2.2, M S_{\mathrm{e}}=0.95\right]$. The results therefore do not suggest that the analyses described above reflect a speed-accuracy trade-off.

\section{Discussion}

We replicated the key results of Experiment 1: There was a significant object-specific priming effect, and this effect was neither reduced nor eliminated by changing the orientation of the object in the target display. Such a result might be interpreted as evidence that the peripheral object preview does not support the construction of a stable representation of object orientation, which cannot, therefore, play a role in the preservation of perceptual continuity. However, the results from the orientation judgment task suggest that subjects do maintain a representation of an object's orientation, and this representation must be specific to that particular object. The data are, therefore, most consistent with an account in which orientation is represented in object files but plays a limited role in supporting object continuity under impoverished preview conditions. One such account will be provided below.

The results of Experiment 3 are in agreement with those of a number of previous studies demonstrating that object orientation is maintained across saccades (e.g., Castelhano \& Henderson, 2005; Henderson \& Hollingworth, 2003; Hollingworth \& Henderson, 2002; Hollingworth, Williams, \& Henderson, 2001). However, the experiment also differs from those studies in important ways. First, the results of the previous studies suggest that performance is dependent on visual object representations in long-term memory. In contrast, the present study focused on object representations that are believed to exist in visual working memory. Apparent limits on the number of episodic object representations that can be maintained at once, for example, suggest that such representations are unlikely to reside within long-term visual memory (e.g., Gordon \& Irwin, 1996, 2000; Henderson, 1994; Henderson \& Anes, 1994).

More significantly, the results of the present study suggest, as those of other studies have not, that the representation of a perceptual feature in memory is not sufficient to ensure that the feature will play a role in preserving object continuity across a saccade. Instead, the results of Experiments 1-3 suggest that the probability that a feature change will disrupt continuity depends on factors, such as eccentricity, that affect the quality of the perceptual representation. 


\section{GENERAL DISCUSSION}

The purpose of the present study was to examine the nature of the object file representations that underlie object continuity, focusing in particular on display properties that affect representation and on how representations change over time. In three experiments, subjects viewed preview displays to which they moved their eyes. Each display contained one or two familiar objects, and when the eyes landed, a single object was presented for subjects to name. Following the reviewing paradigm developed by Kahneman et al. (1992) and adapted by Henderson and his colleagues (e.g., Henderson \& Siefert, 2001), the target matched the preview object in the same or an opposite location or did not match either preview object. This permitted the measurement of object-specific and (in Experiments 1 and 2) nonspecific preview benefits.

There were two key manipulations in Experiment 1. First, on trials on which the target matched one of the preview objects, its orientation was either the same as or different from its preview. Thus, it was possible to examine the role of orientation in preserving object continuity by comparing object-specific preview effects on trials on which the orientation changed with those on trials on which the orientation did not change. Second, the preview-target SOA was manipulated in order to examine how object file representations change over time. It was expected that orientation would play a role in preserving object continuity at short SOAs, but not at long SOAs.

The results of Experiment 1, however, did not confirm this prediction. Although object-specific preview benefits were reduced at long SOAs (presumably reflecting some decay in the representation over time), orientation change did not reduce the benefit at either the short or the long SOA. This suggests that an object's orientation does not play a substantial role in preserving its continuity across saccades.

This conclusion, however, was tempered by the results of Experiment 2, in which the previewed objects were presented closer to fixation. Under such conditions, we replicated Henderson and Siefert's (2001) finding that orientation change disrupts perceptual continuity. The results of Experiments 1 and 2 thus reveal the importance of viewing conditions to visual object representations: Reductions in image quality at large eccentricities appear to be accompanied by a reduced role for object orientation in preserving continuity.

This is not because orientation information is not acquired or represented, however. In Experiment 3, we replicated the short SOA condition from Experiment 1 but added a secondary task on every trial, in which the subjects were required to indicate whether the orientation of the target matched its preview orientation. As in Experiment 1 , changing the object's orientation had no impact on the object-specific preview benefit, suggesting that orientation plays a limited role in preserving object continuity across saccades. Nonetheless, the results from the secondary orientation judgment task confirmed that subjects maintain an object-specific representation of orientation and can use this representation when called upon to do so. Given that the stimuli in Experiment 3 were different from those in Experiments 1 and 2, we cannot argue unequivocally that orientation was acquired and represented in Experiment 1 as it was in Experiment 3. Nevertheless, the results of Experiment 3 provide strong support for an account in which observers acquire and represent information about the orientation of previewed objects but do not rely on that information to maintain continuity when image quality is poor.

In drawing these conclusions, we make a distinction between what an object file represents and how that representation is used to determine object continuity. This is a distinction that has often been overlooked. Typically, when changing an object property does not reduce objectspecific priming, such a result is taken as evidence that the changed property was not part of the object file representation (e.g., Gordon \& Irwin, 1996, 2000; Henderson, 1994). For example, when Gordon and Irwin (2000) found substantial object-specific priming of words by pictures (and vice versa), they concluded that object files must represent abstract object properties (such as identity), rather than physical object features. Similarly, when Henderson found that changing the case of a letter stimulus did not affect transsaccadic priming, he concluded that transsaccadic object file representations do not represent letter case.

The distinction between representations and their use is an important one, however. Recall, for example, that Kahneman et al. (1992) described object continuity as dependent on a series of processes: encoding, correspondence, and a reviewing process that relies on a comparison between currently visible objects and stored object file representations. Implicit in the conclusions drawn by researchers studying object file effects is the assumption that object continuity depends most critically on encoding. Thus, if changing an object feature does not disrupt the object's continuity, the assumption is that the feature was not encoded as part of the object's representation. Another possibility, however, is that the feature does not play a role in the processes that underlie reviewing, even when the feature is part of the object's representation. It may be the case, for example, that object files encode orientation by default, even at large eccentricities, but that continuity is increasingly determined by a comparison of postcategorical information as perceptual representations become less reliable. We argue that the results of Experiments 1-3 are most consistent with the latter explanation.

This conclusion rests on an assumption that performance on the orientation judgment task depends on the same representation that underlies perceptual continuity. This is not obviously correct. Although it must be the case that performance relies on object-specific representations of orientation, such representations may exist in long-term memory, rather than in working memory. Indeed, there is considerable evidence for what Hollingworth and Henderson (2002) have called long-term memory object files, which represent perceptual and conceptual properties of specific objects within a scene. The subjects in Experiment 3 may well have relied on long-term object files. It is important to note, however, that long-term object files 
must be created from episodic object representations in working memory (e.g., Hollingworth \& Henderson, 2002) and that the features included in long-term object files are likely to be those features that were also represented in working memory and that continue to exist within working memory when object continuity is determined. Furthermore, given constraints on episodic representations in working memory (e.g., Henderson \& Anes, 1994), it is unlikely that separate orientation-specific and orientationnonspecific representations are maintained in working memory. A more parsimonious account of the data, therefore, is the one we offer here: that episodic representations include object orientation as a matter of course, but that orientation plays a role in preserving object continuity only when image quality is high.

This account of object continuity, highlighting distinctions between representations and the use of those representations, has resonance in recent theories of change blindness, a marked insensitivity to changes made in scenes. For several years following early demonstrations of change blindness (e.g., Grimes, 1996; Rensink, O'Regan, \& Clark, 1997), dominant accounts of the phenomenon suggested that it reflects a severely impoverished representation of the visual world (e.g., O'Regan, 1992; Rensink, 2000, 2002). Rensink (2000), for example, has suggested that representations of the visual world are limited to the object that is currently the focus of attention. More recently, however, evidence has accumulated that supports an alternative explanation of change blindness (e.g., Angelone, Levin, \& Simons, 2003; Hollingworth, 2003; Hollingworth \& Henderson, 2002; Mitroff, Simons, \& Levin, 2004; Simons, 2000; Zelinsky, 2001, 2003). Mitroff, Simons, and Levin, for example, showed subjects two successive arrays of objects. The arrays were identical, except that, on change trials, one of the objects in the first array was replaced by a new object in the second array. The subjects performed two tasks. First, they answered three 2-alternative forced-choice (2AFC) questions, in which they were shown pairs of objects and were asked to indicate which of the two objects had been present during the trial. One question included the object that was unique to the prechange array, and another question included the object that was unique to the postchange array. Thus, the subjects were required to access their preand postchange representations to carry out the task. Following the 2AFC task, the subjects were asked to report whether or not they had detected a change in the arrays on that trial. The results indicated that change blindness occurred even on those trials on which the 2AFC data suggest that the subjects had access to accurate representations of the pre- and postchange objects. Mitroff, Simons, and Levin concluded that, at least in some cases, change blindness results not from a failure to encode or retain an accurate scene representation, but from a failure to compare pre- and postchange representations.

The results of the present experiments make much the same argument concerning object file representations and their use. The results of Experiments 1 and 3 represent a sort of change blindness, although not as that term is normally used (we do not assume, for example, that the subjects in our experiments were unaware of orientation changes). Instead, it appears that the reviewing process that is responsible for sustaining object continuity is "blind" to changes in object orientation. Because this process relies on episodic object representations, it is as tempting to conclude that such representations are themselves insensitive to orientation as it is to conclude that change blindness necessarily implicates an impoverished scene representation. As in Mitroff, Simons, and Levin (2004), however, we have shown in the present investigation that subjects are able to access object-specific representations of orientation, even when orientation change has no effect on object-specific priming.

Thus, the present results suggest that object orientation is encoded and represented in object files but that it does not always play a role in the comparison that underlies the reviewing process. Although further research into object file retrieval and comparison is necessary, it is likely that the comparison is dominated by postcategorical information (e.g., by representations of object identity). Although comparison of perceptual features may be easier to implement, a consequence of comparing such features is that object continuity will be disrupted as a result of simple transformations that can be expected to occur from object or observer motion. Comparing postcategorical object properties enables a process that is sensitive to changes in an object's identity, while being insensitive to superficial changes in its appearance. Object continuity will be disrupted only when the object's identity changes.

It should be noted, however, that the presumed dominance of postcategorical information may simply reflect the tasks that have typically been used to study perceptual continuity. As in the present study, most studies examining the nature of object file representations have done so within the context of a primary task that required explicit object identification (e.g., Gordon \& Irwin, 1996, 2000; Henderson, 1994; Henderson \& Anes, 1994). If episodic object representations reflect task requirements (e.g., Droll, Hayhoe, Triesch, \& Sullivan, 2005), the results of these studies may overestimate the role of object identity in driving perceptual stability. We are currently carrying out experiments in which task requirements are manipulated in order to examine the influence of such requirements on object file representation and disruptions of perceptual continuity.

The results of the present study should prompt a reconsideration of earlier results showing that object file representations are quite limited. Gordon and Irwin (2000), for example, showed that object continuity is preserved even in the face of an extreme perceptual change, when an object in the preview display is replaced by the object's name in the target display (and vice versa). Gordon and Irwin (2000) concluded that their results provided strong evidence that object files do not represent perceptual object properties. Similarly, Henderson (1994) found that changing a letter's case did not disrupt its continuity and argued that case information was not represented. In light of the present study, we would suggest that those studies show only that perceptual properties or letter case do not play a role in preserving object continuity. Those features 
may well be represented in object files but may not be used as a basis for comparison.

Finally, it may be important to reevaluate the use of the reviewing paradigm developed by Kahneman et al. (1992) for studying object file representation. We believe that it remains a useful paradigm, but one whose use should be limited to cases in which it is most appropriate. For example, one can use the paradigm to study the factors that determine object continuity in general, without drawing conclusions about the role of those factors in the specific processes that support continuity (indeed, Kahneman et al., 1992, used the approach in this limited way). Furthermore, if one finds that changing an object property disrupts continuity, it must be the case that the property is represented in object files. If, however, one finds that changing the object property does not disrupt continuity, one should not conclude that the property is not represented in object files; one can conclude only that it does not play a role in preserving continuity.

\section{Conclusion}

The experiments reported here suggest that object files represent orientation information and that the nature of object file representations does not change over a 1.5-sec interval. However, the results also suggest that the role of perceptual features in preserving object continuity depends on the quality of the representations of those features. Along with a number of studies in the change blindness literature (e.g., Hollingworth, 2003; Mitroff, Simons, \& Levin, 2004), our results provide evidence for a critical distinction between object representations and the use of those representations to support cognitive and perceptual processes.

\section{AUTHOR NOTE}

This project was supported by Grant 1P20 RR020151 from the National Center for Research Resources (NCRR), a component of the National Institutes of Health (NIH). Its contents are solely the responsibility of the authors and do not necessarily represent the official views of the NCRR or the NIH. The project was also supported by the National Science Foundation under Grant BCS-0443998. We thank John Henderson, Greg Zelinsky, and James Hoffman for helpful comments on the manuscript. Correspondence concerning this article may be sent to R. D. Gordon, Department of Psychology, North Dakota State University, Fargo, ND 58105 (e-mail: robert.d.gordon@ndsu.edu).

\section{REFERENCES}

Angelone, B. L., Levin, D. T., \& Simons, D. J. (2003). The roles of representation and comparison failures in change blindness. Perception, 32, 947-962.

AtKins, J. E., Fiser, J., \& JACOBS, R. A. (2001). Experience-dependent visual cue integration based on consistencies between visual and haptic percepts. Vision Research, 41, 449-461.

Castelhano, M. S., \& Henderson, J. M. (2005). Incidental visual memory for objects in scenes. Visual Cognition, 12, 1017-1040.

Cooper, L. A., Schacter, D. L., Ballesteros, S., \& Moore, C. (1992). Priming and recognition of transformed three-dimensional objects: Effects of size and reflection. Journal of Experimental Psychology: Learning, Memory, \& Cognition, 18, 43-57.

Droll, J. A., Hayhoe, M. M., Triesch, J., \& Sullivan, B. T. (2005). Task demands control acquisition and storage of visual information. Journal of Experimental Psychology: Human Perception \& Performance, 31, 1416-1438.
Feldman, J. A. (1985). Four frames suffice: A provisional model of vision and space. Behavioral \& Brain Sciences, 8, 265-289.

Gajewski, D. A., \& Henderson, J. M. (2005). The role of saccade targeting in the transsaccadic integration of object types and tokens. Journal of Experimental Psychology: Human Perception \& Performance, 31, 820-830.

Germeys, F., De Graef, P., \& Verfaillie, K. (2002). Transsaccadic perception of saccade target and flanker objects. Journal of Experimental Psychology: Human Perception \& Performance, 28, 868-883.

Gordon, R. D., \& IRWIN, D. E. (1996). What's in an object file? Evidence from priming studies. Perception \& Psychophysics, 58, 1260-1277.

Gordon, R. D., \& IRWIN, D. E. (2000). The role of physical and conceptual properties in preserving object continuity. Journal of Experimental Psychology: Learning, Memory, \& Cognition, 26, 136-150.

Grimes, J. (1996). On the failure to detect changes in scenes across saccades. In K. Akins (Ed.), Perception (Vol. 2, pp. 89-110). New York: Oxford University Press.

Henderson, J. M. (1994). Two representational systems in dynamic visual identification. Journal of Experimental Psychology: General, 123, 410-426.

Henderson, J. M., \& Anes, M. D. (1994). Roles of object-file review and type priming in visual identification within and across eye fixations. Journal of Experimental Psychology: Human Perception \& Performance, 20, 826-839.

Henderson, J. M., \& Hollingworth, A. (2003). Eye movements and visual memory: Detecting changes to saccade targets in scenes. Perception \& Psychophysics, 65, 58-71.

Henderson, J. M., \& Siefert, A. B. C. (2001). Types and tokens in transsaccadic object identification: Effects of spatial position and left-right orientation. Psychonomic Bulletin \& Review, 8, 753-760.

Hollingworth, A. (2003). Failures of retrieval and comparison constrain change detection in natural scenes. Journal of Experimental Psychology: Human Perception \& Performance, 29, 388-403.

Hollingworth, A., \& Henderson, J. M. (2002). Accurate visual memory for previously attended objects in natural scenes. Journal of Experimental Psychology: Human Perception \& Performance, 28, 113-136.

Hollingworth, A., Williams, C. C., \& Henderson, J. M. (2001). To see and remember: Visually specific information is retained in memory from previously attended objects in natural scenes. Psychonomic Bulletin \& Review, 8, 761-768.

IRWIN, D. E. (1991). Information integration across saccadic eye movements. Cognitive Psychology, 23, 420-456.

IRWIN, D. E. (1992). Memory for position and identity across eye movements. Journal of Experimental Psychology: Learning, Memory, \& Cognition, 18, 307-317.

IRWIN, D. E. (1996). Integrating information across saccadic eye movements. Current Directions in Psychological Science, 5, 94-100.

IRWIN, D. E., \& ANDREWS, R. V. (1996). Integration and accumulation of information across saccadic eye movements. In T. Inui \& J. L. McClelland (Eds.), Attention and performance XVI: Information integration in perception and communication (pp. 125-156). Cambridge, MA: MIT Press, Bradford Books.

IRWIN, D. E., \& GoRdon, R. D. (1998). Eye movements, attention, and transsaccadic memory. Visual Cognition, 5, 127-155.

IrWin, D. E., \& Zelinsky, G. J. (2002). Eye movements and scene perception: Memory for things observed. Perception \& Psychophysics, 64, 882-895.

Kahneman, D., \& Treisman, A. (1984). Changing views of attention and automaticity. In R. Parasuraman \& R. Davies (Eds.), Varieties of attention (pp. 29-61). Cambridge, MA: MIT Press.

Kahneman, D., Treisman, A., \& Gibbs, B. J. (1992). The reviewing of object files: Object-specific integration of information. Cognitive Psychology, 24, 175-219.

Matin, E. (1974). Saccadic suppression: A review and an analysis. Psychological Bulletin, 81, 899-917.

McConkie, G. W., \& RaYner, K. (1976). Identifying the span of the effective stimulus in reading: Literature review and theories of reading. In H. Singer \& R. B. Ruddell (Eds.), Theoretical models and processes of reading (pp. 137-162). Newark, DE: International Reading Association.

Mitroff, S. R., Scholl, B. J., \& WynN, K. (2004). Divide and conquer: 
How object files adapt when a persisting object splits into two. Psychological Science, 15, 420-425.

MitrofF, S. R., Simons, D. J., \& Levin, D. T. (2004). Nothing compares 2 views: Change blindness can occur despite preserved access to the changed information. Perception \& Psychophysics, 66, 1268-1281.

Noles, N. S., Scholl, B. J., \& Mitroff, S. R. (2005). The persistence of object file representations. Perception \& Psychophysics, 67, 324-334.

O'Regan, J. K. (1992). Solving the "real" mysteries of visual perception: The world as an outside memory. Canadian Journal of Psychology, 46, 461-488.

Rensink, R. A. (2000). The dynamic representation of scenes. Visual Cognition, 7, 17-42.

Rensink, R. A. (2002). Change detection. Annual Review of Psychology, 53, 245-277.

Rensink, R. A., O'Regan, J. K., \& Clark, J. J. (1997). To see or not to see: The need for attention to perceive changes in scenes. Psychological Science, 8, 368-373.

Richard, A. M., Hollingworth, A., \& LuCK, S. J. (2006). Testing an object file theory of object correspondence across saccades [Abstract]. Journal of Vision, 6, 486a. Available at journalofvision.org/6/6/486/, doi: $10.1167 / 6.6 .486$.
Simons, D. J. (2000). Current approaches to change blindness. Visual Cognition, 7, 1-15.

Snodgrass, J. G., \& Vanderwart, M. (1980). A standardized set of 260 pictures: Norms for agreement, image agreement, familiarity, and visual complexity. Journal of Experimental Psychology: Human Learning \& Memory, 6, 174-215.

Treisman, A., Kahneman, D., \& Burkell, J. (1983). Perceptual objects and the cost of filtering. Perception \& Psychophysics, 33, 527-532.

Verfaillie, K., \& De Graef, P. (2000). Transsaccadic memory for position and orientation of saccade source and target. Journal of Experimental Psychology: Human Perception \& Performance, 26, 1243-1259.

ZeLINSKY, G. J. (2001). Eye movements during change detection: Implications for search constraints, memory limitations, and scanning strategies. Perception \& Psychophysics, 63, 209-225.

ZeLINSKY, G. J. (2003). Detecting changes between real-world objects using spatiochromatic filters. Psychonomic Bulletin \& Review, 10, 533-555.

(Manuscript received September 8, 2006; revision accepted for publication December 11, 2007.) 